\title{
FOOD INTOLERANCE: INDUSTRIAL PRODUCTS OR NATURAL FOODS?
}

\section{Barbara Paolini}

\author{
Department of Dietetics and Clinical Nutrition \\ AOU Senese, Policlinico Santa Maria alle Scotte-Siena, Italy
}

\begin{abstract}
Food intolerances are often considered responsible for abdominal symptoms, like bloating, pain and alterations of bowel movements after the assumption of certain foods. Restrictive diets are very popular and they are based on food intolerance not scientifically validated diagnostic tests. Inappropriate use of these tests increases the possibility of false positive, with useless dietetic restrictions, risk of malnutrition and reduced life quality. Exclusion diets must be monitored and managed by specialists to avoid nutritional risks. They increase the phenomenon of "diet industry" and they represent a direct cost for the patients and an indirect cost for the public health, because the therapy is inadequate for the needs. The industry created a big range of products for people with food intolerances for example to lactose, gluten, however they have a bigger content of fat and calories in comparison with the classic products in order to improve their taste. Processed products contain substances like emulsifiers, stabilizers and acidity correctors so they have a worse quality. It is important to not exclude foods from our diet unless a food intolerance is diagnosed by validated tests, in addiction to this, considering that the diet remains the only life-style therapy for many intolerances, it is crucial to prefer not processed products and naturally gluten or lactose free products.
\end{abstract}

Key words: food intolerance, diet industry, gluten, diet.

Food intolerance has been the topic of discussion for long time because of the confusion that occurs in most of the population by attributing to them the responsibility for abdominal disorders such as swelling, bloating, pain and/or alteration of the bowel habits after the assumption of certain foods, or more often weight increasing or metabolic diseases. Recently, there was an enormous diffusion, especially on the media (web and social networks), of restrictive diets based on diagnostic tests of "food intolerance" performed on different biological samples (blood, saliva, hair), as detoxifying remedies to the body. Some doctors in ancient Greece described cases of food adverse reactions but recent scientific studies have dated to the early 20th century.

The allergy is instead an expression of an abnormal answer of the immune system against harmless food, but recognized as harmful in some predisposed subjects. Typical symptoms of food allergies occur a few minutes or a few hours after the intake of the responsible food. According to epidemiological data, food allergies (AA) affect $5 \%$ of children under 3 years and about $4 \%$ of the adult population [1]. In the general population, the concept of "food allergy" is widespread (about $20 \%$ of the population feels that they are affected by food allergies). "perceived" food allergies, however, are not always real: self-evaluation data, ranging between $12.4 \%$ and $25 \%$, are confirmed by the oral provocation test (TPO) only in $1.5-3.5 \%$ of the cases $[2,3]$.

The available diagnostic tests for food allergy are: Skin tests (prick test, prick by prick, patch test); Serological tests for total IgE (PRIST) and specific (via ImmunoCAP or RAST) research; Molecular diagnostics; Double-blind Oral Provocation Test (TPO) versus placebo [4].

The definition of food intolerance is related to adverse reactions to foods occurring from a few hours to a few days after the intake, and unlike food allergies, they are not related to the production of a particular class of IgE antibodies (responsible for allergic reactions).

Symptoms include chronic diarrhea, abdominal swelling, vomiting, meteorism, abdominal cramps, migraine, but sometimes they overlap with those of a food allergy (such as urticaria, itching or tongue).

Food intolerances include: i) Enzymatic reactions, determined by the absence or deficiency of enzymes necessary to metabolize some substrates (e.g. lactose intolerance); ii) pharmacological reactions, responses

\section{Correspondence to:}

Barbara Paolini

Department of Dietetics and Clinical Nutrition - AOU Senese, Policlinico Santa Maria alle Scotte-Siena, Strada delle Scotte 1, 53100 Siena, Italy

Tel.: +39.3337703771.

E-mail: barbara-paolini@libero.it 
to pharmacologically active food components, such as vasoactive amines (e.g. tyramine, histamine and caffeine) contained in fish, chocolate and fermented products, or substances added to foods (colorants, additives..); iii) indefinite responses, like psychological or neurological responses (food aversion or rhinorrhoea) [5].

The diagnostic approach in suspecting a food intolerance is primarily based on anamnesis.

Currently validated tests for diagnosis of food intolerances are: breath test for lactose intolerance, specific antibody assay, and endoscopic examination for gluten intolerance. For the diagnosis of pharmacological intolerances, the only approach is anamnestic but for those linked to undefined mechanisms it is possible to carry out the provocation test with the administration of the suspected additive [6].

Recently, however, the speculative fashion on intolerance led to the birth of unvalidated and expensive tests without any scientific evidence, which may delay a proper diagnosis and lead to exclusion of well-tolerated foods.

Unvalidated tests are: IGg4 assay, cytotoxic test, Alcat test, electrical tests (vega-test, Voll electroacupuncture, bioscreening, biostrengt test, sarm test, moratest), kinesiologic test, hair analysis, iridology, Bioresonance, pulse test, ear cardiac reflex. The inappropriate use of these tests only increases the likelihood of false positives, resulting in unnecessary dietary restrictions and reduced quality of life [7].

Exclusion diets not adequately managed and monitored by a qualified healthcare professional can lead to nutritional risk and deficiencies, and poor growth and malnutrition in children, and they can increase the phenomenon of the "diet industry" and, moreover they cause a direct cost for patients / users and indirect for the National Healthcare System, as the therapeutic response is inadequate to the needs. When taking an exclusion diet, even for a single food or food group, clear nutrition informations must be provided in order to ensure, first of all, an adequate caloric intake as well as for macro and micronutrients. Adequate follow-up is essential in order to evaluate compliance with diet, early detection of nutritional deficiencies and, in children, to ensure regular growth [8].

Lactose intolerance is caused by a lactase deficiency. Non-absorption of lactose causes diarrhea, cramps and abdominal pain, abdominal tension, flatulence. Symptoms arise within a few hours of lactose ingestion and they are extremely heterogeneous. The amount of lactose responsible for the clinical symptoms is quite variable. Diet therapy consists in exclusion of milk and dietary sources, however, lactose is contained in many other products (baked goods, sausages, etc.). Many lactose-free foods are available on the market, allowing these patients to maintain their usual eating habits. Nutritional properties do not differ from lactose products $[9,10]$.

Celiac disease is a permanent intolerance to gluten that causes, in genetically predisposed subjects, a chronic inflammatory reaction of the small intestine mucosa resulting in global malabsorption for progressive flattening up to the complete disappearance of intestinal wildlife. The only therapy is a strictly gluten-free diet that is to be followed throughout life because, whatever clinical expression is, it is a permanent condition. As with lactose, even in celiac disease, the industry today has made available a wide range of gluten-free products, but in order to improve its palatability, percentage fat and total calories are more than classic products. Considering that diet remains the only life-style therapy, it is crucial that the diet prefers naturally gluten-free (cereal) products [11].

\section{REFERENCES}

1. Bégin P, Nadeau KC. Diagnosis of Food Allergy. Pediatr Ann 2013:42:102-9.

2. Boyce JA, Assa'ad A, Burks AW, et al. Guidelines for the diagnosis and management of food allergy in the United States: report of the NAID-sponsored expert panel. J Allergy Clin Immunol 2010;126:S1-S58.

3. Boyce JA, Assa'ad A, Burks AW, et al. Guidelines for the diagnosis and management of food allergy in the United States: summary of the NIAID-sponsored expert panel report. Nutr Res 2011;31:61-75.

4. SIAP, SIPPS. Guida Pratica sulle Allergie. Editem. 2015.

5. SIAAIC-AAITO-SIAIP, FNOMCeO. Allergie e intolleranze alimentari. Documento condiviso. Febbraio 2016, Ed. Zadig.

6. Senna G, Bonadonna P, Schiappoli $M$, et al. Pattern of use and diagnostic value of complementary/alternative tests for adverse reactions to food. Allergy 2005;60:1216-7.

7. Stapel SO, Asero R, Ballmer-Weber BK, et al. Position Paper: Testing for IgG4 against foods is not recommended as a diagnostic tool: EAACI Task Force Report. Allergy 2008:63:793-6.

8. SIAIP. Position paper su Choosing Wisely. Rivista di Immunologia e Allergologia Pediatrica 2014; 1:1-6. Ed. Pacini.

9. Misselwitz, B, Pohl D, Frühauf $H$, et al. Lactose malabsorption and intolerance: pathogenesis, diagnosis and treatment. United European Gastroenterol J 2013;1:151-9.

10. Gasbarrini A, Corazza GR, Gasbarrini G, et al. 1st Rome H2Breath Testing Consensus Conference Working Group. Methodology and indications of H2-breath testing in gastrointestinal diseases: the Rome Consensus Conference. Aliment Pharmacol Ther 2009;29:1-49.

11. Società Italiana di Diabetologia (SID) Associazione Italiana di Dietetica e Nutrizione Clinica (ADI) Associazione Medici Diabetologi (AMD) Associazione Nazionale Dietisti (ANDID) Società Italiana di Nutrizione Umana (SINU) Società Italiana di Nutrizione Pediatrica (SINUPE) Società Italiana dell'Obesità (SIO). Position Statement su Allergie, intolleranze alimentari e terapia nutrizionale dell'obesità e delle malattie metaboliche. 2016. 